\title{
PHASE EQUILIBRIA OF CARBON DIOXIDE AND METHANE GAS-HYDRATES PREDICTED WITH THE MODIFIED ANALYTICAL S-L-V EQUATION OF STATE
}

\begin{abstract}
Václav VINŠ ${ }^{1}$, Andreas JÄGER ${ }^{2}$, Jan HRUBÝ ${ }^{1}$, Roland SPAN ${ }^{2}$
Abstract: Gas-hydrates (clathrates) are non-stoichiometric crystallized solutions of gas molecules in the metastable water lattice. Two or more components are associated without ordinary chemical union but through complete enclosure of gas molecules in a framework of water molecules linked together by hydrogen bonds. The clathrates are important in the following applications: the pipeline blockage in natural gas industry, potential energy source in the form of natural hydrates present in ocean bottom, and the $\mathrm{CO}_{2}$ separation and storage. In this study, we have modified an analytical solid-liquid-vapor equation of state (EoS) [A. Yokozeki, Fluid Phase Equil. 222-223 (2004)] to improve its ability for modeling the phase equilibria of clathrates. The EoS can predict the formation conditions for $\mathrm{CO}_{2-}$ and $\mathrm{CH}_{4}$-hydrates. It will be used as an initial estimate for a more complicated hydrate model based on the fundamental EoSs for fluid phases.
\end{abstract}

\section{INTRODUCTION TO GAS-HYDRATES}

Clathrates of natural gas, commonly called gas-hydrates, are non-stoichiometric solid solutions of a low molecular weight gas and water [1]. The gas-hydrates are becoming important in many areas of human activities. Originally, the gas-hydrates were investigated due to the pipeline blockage in the natural gas industry. With increasing energy consumption, decreasing reserves of fossil fuels, and climate changes, the gashydrates are being investigated for two following applications: potential energy source in the form of natural hydrates present in the ocean bottom, and the carbon dioxide separation and storage [2].

The clathrate is a solid crystallized solution of the gaseous component in the thermodynamically metastable water lattice. In gas-hydrates, the gas molecule, called the "guest", is situated in the cavity, referred also as the "cage" or "host", formed by a framework of water molecules linked together by hydrogen bonds. The guest-host interactions are realized through van der Waals type dispersion forces. The clathrate becomes thermodynamically stable under given temperature and pressure if a certain fraction of cavities is occupied by the guest molecules. The guest occupancy of the cavities stabilizes the empty water lattice.

\footnotetext{
${ }^{1}$ Institute of Thermomechanics AS CR, v. v. i., Dolejškova 1402/5, 18200 Prague 8, Czech Republic (vins.vaclav@seznam.cz, hruby@it.cas.cz)

2 Institute for Thermodynamics, Ruhr-Universität Bochum, Universitätsstr. 150, 44780 Bochum, Germany (a.jaeger@thermo.ruhr-uni-bochum.de, roland.span@thermo.rub.de)
}

This is an Open Access article distributed under the terms of the Creative Commons Attribution License 2.0, which permits unrestricted use, distribution, and reproduction in any medium, provided the original work is properly cited. 
Most of the hydrate models are based on the theory by van der Waals and Platteeuw (vdWP) [1] combining the statistical thermodynamics with the classical thermodynamics. The vdWP theory was further improved by Parrish and Prausnitz [3] for practical calculations of the hydrate formation conditions. Temporary hydrate models such as that by Lee and Holder [4], Klauda et al. [5,6], Ballard and Sloan [7,8], managed to avoid most of the limiting assumptions of the original models by van der Waals and Platteeuw [1] and Parrish and Prausnitz [3]. However application of these models [4]-[8] is still rather complicated and requires advanced computational ability.

On the other hand, Yokozeki [9] developed a simple analytical equation of state (EoS) that can provide qualitatively good estimate for the solid-liquid-vapor (S-L-V) equilibria. This EoS can simultaneously model all three phases and can be extended to simple mixtures such as $\mathrm{CO}_{2}+\mathrm{CH}_{4}$. In the following two studies $[10,11]$, Yokozeki demonstrated that his EoS may also be used for gas-hydrates modeling. The S-L-V EoS provided qualitatively good prediction for $\mathrm{CH}_{4}-$ and $\mathrm{CO}_{2}$-hydrate formation conditions. The Yokozeki's EoS is therefore a relatively simple alternative to much more complicated hydrate models based on the vdWP theory.

In this study, we modified the original Yokozeki's [9]-[11] S-L-V EoS to improve its predictive ability for $\mathrm{CH}_{4}$ - and $\mathrm{CO}_{2}$-hydrate phase equilibria. Moreover, there were found several small discrepancies in the Yokozeki's articles [9]-[11] which we also tried to fix in this work. The modified S-L-V EoS models quite accurately the formation conditions for both gas-hydrates over relatively wide ranges of temperature and pressure. It is planned to use this EoS as an initial estimate for a more complicated hydrate model based on the vdWP theory combined with the fundamental EoSs for fluid phases [12]-[14] and for the solid phase of pure components, i.e. water ice Ih [15] and dry ice [16].

\section{ANALYTICAL EqUATION OF STATE FOR SOLID-LIQUID-VAPOR}

Yokozeki's [9] analytical EoS represents a physically reasonable extension of the original van der Waals [17] fluid EoS. The final form of the S-L-V EoS is given as follows

$$
p=\frac{R T}{v-b}\left(\frac{v-d}{v-c}\right)-\frac{a}{v^{2}}
$$

with volumetric parameters $0<b<d<c$. Figure 1 shows a $p$ - $\log (v)$ diagram for carbon dioxide obtained from the S-L-V EoS (1). The EoS is compared with the cubic PengRobinson [18] EoS for fluid phases.

Reduced parameters of the S-L-V EoS are defined in the following manner

$$
a_{\mathrm{r}}=a \frac{p_{\mathrm{c}}}{\left(R T_{\mathrm{c}}\right)^{2}}, b_{\mathrm{r}}=b \frac{p_{\mathrm{c}}}{Z_{\mathrm{c}} R T_{\mathrm{c}}}, \quad c_{\mathrm{r}}=c \frac{p_{\mathrm{c}}}{Z_{\mathrm{c}} R T_{\mathrm{c}}}, d_{\mathrm{r}}=d \frac{p_{\mathrm{c}}}{Z_{\mathrm{c}} R T_{\mathrm{c}}},
$$

where the attraction parameter $a_{\mathrm{r}}$ and the volumetric parameter $b_{\mathrm{r}}$ are considered temperature dependent

$$
\begin{gathered}
a_{\mathrm{r}}(T)=a_{0}+a_{1} T / T_{\mathrm{c}} \exp \left[-a_{2}\left(T / T_{\mathrm{c}}\right)^{n}\right] \\
b_{\mathrm{r}}(T)=b_{0}+b_{1} \exp \left[-b_{2}\left(T / T_{\mathrm{c}}\right)^{m}\right] .
\end{gathered}
$$

In the modified S-L-V EoS, the pure component parameters $a$ to $d$ were taken from Yokozeki [9] for $\mathrm{CO}_{2}$ and from Yokozeki [11] for $\mathrm{H}_{2} \mathrm{O}$ and $\mathrm{CH}_{4}$. Values for the reduced parameters, coefficients, and exponents in equations (2) to (4) can be found in the original articles by Yokozeki $[9,11]$. 


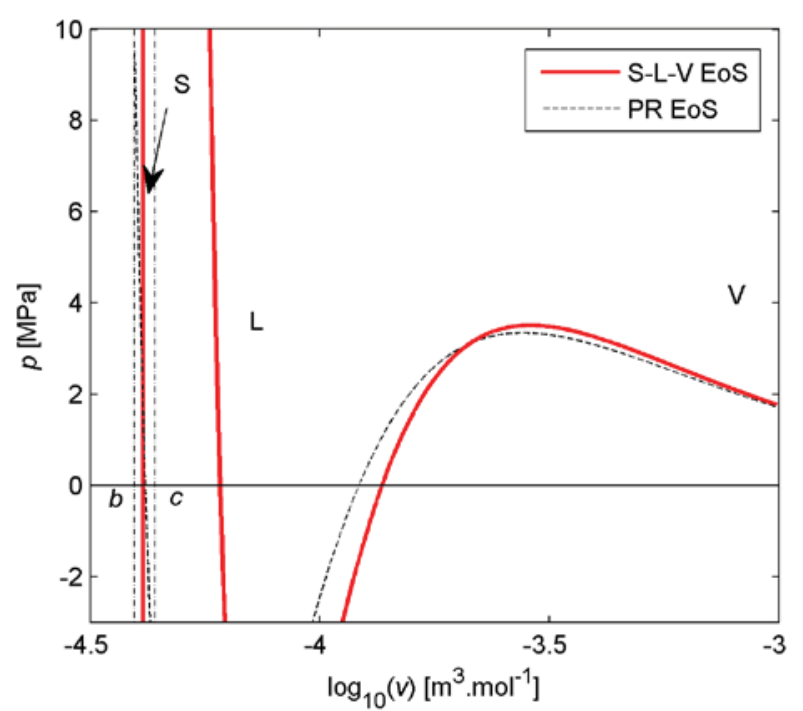

Figure 1: Schematic $p v$ diagram for $\mathrm{CO}_{2}$ calculated from the S-L-V EoS (1) and the Peng-Robinson EoS

Table 1 compares the temperature and pressure at the triple point calculated from the SL-V EoS for vapor (V) - liquid (L) - solid (I) phase equilibria with the tabulated values. The water parameters provided in [10] must contain some kind of inaccuracy as they did not result in the satisfactory triple point. The calculated temperature $258.23 \mathrm{~K}$ and pressure $0.186 \mathrm{kPa}$ differed strongly from the tabulated values in this case.

\begin{tabular}{ccccccc}
\hline & $T[\mathrm{~K}]$ & $T(\mathrm{EoS})[\mathrm{K}]$ & $\mathrm{d} T[\%]$ & $p[\mathrm{kPa}]$ & $p(\mathrm{EoS})[\mathrm{kPa}]$ & $\mathrm{d} p[\%]$ \\
\hline $\mathrm{CH}_{4}$ & 90.694 & 90.342 & 0.389 & 11.696 & 11.679 & 0.142 \\
$\mathrm{CO}_{2}$ & 216.592 & 216.531 & 0.028 & 517.960 & 516.962 & 0.193 \\
$\mathrm{H}_{2} \mathrm{O}$ & 273.160 & 273.400 & -0.088 & 0.612 & 0.621 & -1.585 \\
\hline
\end{tabular}

\section{Table 1: Comparison of the triple point conditions for pure substances calculated by the analytical S-L-V EoS with the tabulated values}

Mixtures can be treated with a help of the van der Waals / Lorentz-Berthelot mixing rules [9] in the S-L-V EoS. The mixture parameters $a$ to $d$ can be calculated from the mole fractions $x_{i}$ in the following way

$$
a=\sum_{i}^{N} \sum_{j}^{N} \sqrt{a_{i} a_{j}}\left(1-k_{i j}\right) x_{i} x_{j}, b=\sum_{i=1}^{N} b_{i} x_{i}, c=\sum_{i=1}^{N} c_{i} x_{i}, d=\sum_{i=1}^{N} d_{i} x_{i} .
$$

In equation (5), $N=2$ for a binary mixture and $k_{i j}$ stands for the binary interaction parameter. For simple mixtures of "normal" compounds such as $\mathrm{CO}_{2}+\mathrm{CH}_{4}$, the binary interaction parameter may be considered constant, i.e. independent of composition or temperature [9]. However as Yokozeki showed in his other studies $[10,11]$, the aqueous solutions cannot be sufficiently modeled with constant $k_{i j}$. For mixtures containing water, the binary interaction parameter $k_{i j}$ has to be considered composition dependent. Therefore in equation (5), $k_{i j}$ has to be replaced with the variable interaction parameter $K_{i j}$ given as follows 


$$
K_{i j}=\frac{k_{i j} k_{j i}\left(x_{i}+x_{j}\right)}{k_{i j} x_{i}+k_{j i} x_{j}} .
$$

We note that the $K_{i j}$ parameter considered in this study has different component-indices in the denominator than the parameter employed by Yokozeki $[10,11]$. Definition of $K_{i j}$ by equation (6) should be more consistent with the usual form of the van Laar's mixing rule [19].

\section{Phase Equilibrium of a Binary Mixture}

Phase equilibrium of a multicomponent system is generally defined by equality of chemical potentials of each component in all phases present. This condition can be transformed to equality of fugacities which is a more common case in the EoS-based calculations. At a constant pressure, the fugacity-based equilibrium condition can be defined only in terms of the fugacity coefficients $\phi_{i}$ and the mole fractions $x_{i}$

$$
x_{i}^{\mathrm{H}} \phi_{i}^{\mathrm{H}}=x_{i}^{\mathrm{L}_{2}} \phi_{i}^{\mathrm{L}_{2}}=x_{i}^{\mathrm{V}} \phi_{i}^{\mathrm{V}} \text {. }
$$

Equation (7) is an example for the three phase equilibrium of the hydrate $(\mathrm{H})$, water-rich liquid $\left(L_{2}\right)$, and vapor phases. In our calculations, we would like to use the analytical S-L$\checkmark$ EoS (1) as an initial estimate for the mole fractions at the given system temperature and pressure. Therefore, we directly solve the equilibrium condition given by equation (7) instead of using the graphically-based "common tangent" method proposed by Yokozeki [11]. In such a case, the equilibrium condition (7) is solved by a multidimensional Newton-Raphson optimization method applied on the following set of equations

$$
\begin{aligned}
& x_{1}^{\mathrm{L}_{2}} \phi_{1}^{\mathrm{L}_{2}}-x_{1}^{\mathrm{H}} \phi_{1}^{\mathrm{H}}=0 \\
& x_{2}^{\mathrm{L}_{2}} \phi_{2}^{\mathrm{L}_{2}}-x_{2}^{\mathrm{H}} \phi_{2}^{\mathrm{H}}=0 \\
& x_{1}^{\mathrm{V}} \phi_{1}^{\mathrm{V}}-x_{1}^{\mathrm{H}} \phi_{1}^{\mathrm{H}}=0 \\
& x_{2}^{\mathrm{V}} \phi_{2}^{\mathrm{V}}-x_{2}^{\mathrm{H}} \phi_{2}^{\mathrm{H}}=0
\end{aligned}
$$

The pressure is usually set as the independent variable and the temperature and the mole fractions $x_{1}^{\mathrm{L}_{2}}, x_{1}^{\mathrm{H}}$, and $x_{1}^{\mathrm{V}}$ are found as the unknown quantities. Mole fractions of the second component in a binary mixture are obtained from the simple condition $x_{2}^{\alpha}=1-x_{1}^{\alpha}$. According to Yokozeki [9], the fugacity coefficient in equation (8) can be determined as follows

$$
\begin{aligned}
\ln \phi_{i} & =\frac{1}{b-c}\left[c \ln \left|1-\frac{c}{v}\right|-b \ln \left(1-\frac{b}{v}\right)\right]-\frac{c_{i}(b-d)+b_{i}(d-c)+(b-c)\left(d-d_{i}\right)}{(b-c)^{2}} \ln \left|\frac{v-c}{v-b}\right| \\
& -\frac{1}{b-c}\left[\frac{c_{i}(c-d)}{v-c}+\frac{b_{i}(d-b)}{v-b}\right]-\frac{\partial a}{\partial x_{i}} \frac{1}{v R T}-\ln Z
\end{aligned}
$$

where the composition derivative of the attraction parameter $a$ can be derived from equations (5) and (6) in the following way

$$
\frac{\partial a}{\partial x_{i}}=2 \sum_{j}^{2} \sqrt{a_{i} a_{j}}\left(1-K_{i j}\right) x_{j}-\sqrt{a_{i} a_{j}} x_{i} x_{j} \frac{k_{i j} k_{j i}\left(k_{i j} x_{i}+k_{j i} x_{j}\right)-k_{i j}^{2} k_{j i}\left(x_{i}+x_{j}\right)}{\left(k_{i j} x_{i}+k_{j i} x_{j}\right)^{2}} .
$$

To assure good convergence of the phase equilibrium algorithm and to improve its numerical accuracy, the S-L-V EoS (1) was reduced according to the first component critical point (subscript $\mathrm{c} 1$ ), i.e. 


$$
p^{\prime}=\frac{T^{\prime}}{Z_{\mathrm{cl}}\left(v^{\prime}-b^{\prime}\right)} \frac{v^{\prime}-d^{\prime}}{v^{\prime}-c^{\prime}}-\frac{a^{\prime}}{v^{\prime 2} Z_{\mathrm{cl}}^{2}} .
$$

The reduced quantities and the mixture parameters are defined as follows

$$
\begin{gathered}
p^{\prime}=\frac{p}{p_{c 1}}, v=v^{\prime} \frac{Z_{c 1} R T_{c 1}}{p_{c 1}}, T^{\prime}=\frac{T}{T_{c 1}}, \\
a=a^{\prime} \frac{\left(R T_{\mathrm{cl}}\right)^{2}}{p_{\mathrm{c} 1}}, b=b^{\prime} \frac{Z_{\mathrm{c} 1} R T_{\mathrm{c} 1}}{p_{\mathrm{c} 1}}, c=c^{\prime} \frac{Z_{\mathrm{cl}} R T_{\mathrm{c} 1}}{p_{\mathrm{c} 1}}, d=d^{\prime} \frac{Z_{\mathrm{cl}} R T_{\mathrm{cl}}}{p_{\mathrm{c} 1}} .
\end{gathered}
$$

The pure-component attraction parameters reduced according to the first component's critical point are given in the following manner

$$
a_{1 \mathrm{r}}^{\prime}=a_{1 \mathrm{r}}, a_{2 \mathrm{r}}^{\prime}=a_{2 \mathrm{r}} \frac{T_{\mathrm{c} 2}^{2} p_{\mathrm{c} 1}}{T_{\mathrm{c} 1}^{2} p_{\mathrm{c} 2}} .
$$

The volumetric parameters for a pure component reduced according to the first component critical point are defined as follows

$$
e_{1 r}^{\prime}=e_{1 r}, e_{2 r}^{\prime}=e_{2 r} \frac{Z_{c 2} T_{c 2} p_{c 1}}{Z_{c 1} T_{c 1} p_{c 2}} \text { with } e=b, c, d \text {. }
$$

In equations (14) and (15), the reduced parameters of a pure component $a_{r}, b_{r}, c_{r}$, and $d_{r}$ are given by equation (2). The fugacity coefficient can be calculated from the reduced EoS (11) in the following way

$$
\begin{aligned}
\ln \phi_{i}= & \frac{1}{b^{\prime}-c^{\prime}}\left[c^{\prime} \ln \left|1-\frac{c^{\prime}}{v^{\prime}}\right|-b^{\prime} \ln \left(1-\frac{b^{\prime}}{v^{\prime}}\right)\right]-\frac{c_{i \mathrm{r}}^{\prime}\left(b^{\prime}-d^{\prime}\right)+b_{i \mathrm{r}}^{\prime}\left(d^{\prime}-c^{\prime}\right)+\left(b^{\prime}-c^{\prime}\right)\left(d^{\prime}-d_{i \mathrm{r}}^{\prime}\right)}{\left(b^{\prime}-c^{\prime}\right)^{2}} . \\
& \cdot \ln \left|\frac{v^{\prime}-c^{\prime}}{v^{\prime}-b^{\prime}}\right|-\frac{1}{b^{\prime}-c^{\prime}}\left[\frac{c_{i \mathrm{r}}^{\prime}\left(c^{\prime}-d^{\prime}\right)}{v^{\prime}-c^{\prime}}+\frac{b_{i \mathrm{r}}^{\prime}\left(d^{\prime}-b^{\prime}\right)}{v^{\prime}-b^{\prime}}\right]-\frac{\partial a^{\prime}}{\partial x_{i}} \frac{1}{v^{\prime} T^{\prime} Z_{\mathrm{c} 1}}-\ln \left(\frac{p^{\prime} v^{\prime}}{T^{\prime}} Z_{\mathrm{c} 1}\right)
\end{aligned}
$$

The composition derivative of parameter $a$, i.e. $\partial a / \partial x_{i}$, can be transformed to its reduced form $\partial a^{\prime} / \partial x_{i}$, required in equation (16), by replacing the pure component parameters $a_{i}$ and $a_{j}$ in equation (10) with their reduced values $a_{i \mathrm{r}}^{\prime}$ and $a_{j \mathrm{r}}^{\prime}$ given by equation (14).

The phase equilibrium algorithm based on the set of equations similar to equation (8) can be used for two- and three-phase equilibria of a pure component or for two-, three-, and four-phase equilibria of a binary mixture.

\section{Hydrate Phase Model}

The analytical S-L-V EoS can be relatively simply used for normal mixtures, forming just one solid phase, as demonstrated by Yokozeki [9] for a binary mixture of $\mathrm{CO}_{2}+\mathrm{CH}_{4}$. However the application on the hydrate forming systems requires some additional modifications. The S-L-V EoS must provide a description of two different solid phases; namely the pure-component ice, e.g. water ice Ih or dry ice of $\mathrm{CO}_{2}$, and the solid twocomponent solution, i.e. the gas-hydrate. Yokozeki introduced empirical correction coefficients describing the difference between the pure-component ice phase and the hydrate phase. The volumetric parameter $b$ of water was corrected in his $\mathrm{CO}_{2}-$ and $\mathrm{CH}_{4}-$ hydrate models. In the first case, the parameter $b$ was reduced for the water ice [10], while in the second case the parameter $b$ was increased for the water in the hydrate phase [11]. Unfortunately, we found that neither of these two approaches provided satisfactory results. The option of considering two different types of water ice, i.e. one for the pure water and one for the binary mixture forming gas-hydrate, is not appropriate in 
our point of view. On the other hand, the second option with increased volume of the hydrate phase compared to the water ice seems quite reasonable. Nevertheless, we detected some errors in the phase equilibrium calculated with such modified hydrate phase. The "common tangent" method used for the verification of our results did not work properly for the hydrate phase in this case.

In our gas-hydrate model, we concluded with another option proposed by Yokozeki [11] for the hydrate phase modification. Instead of correcting the volumetric parameter $b$, we decreased the attraction parameter $a$ for the gas-hydrate. The interactions of molecules in the hydrate phase are weaker than in the water ice. Consequently, the attraction parameter $a$ had to be lowered in the following manner

$$
a_{\mathrm{H}_{2} \mathrm{O}}(\text { hydrate })=a_{\mathrm{H}_{2} \mathrm{O}}(\text { ice }) / c_{a} \text {, with } c_{a}=1.025 \text {. }
$$

In equation (17), $c_{a}$ is an empirical coefficient whose value was found according to the fact that the gas amount in both the $\mathrm{CO}_{2}$ - and $\mathrm{CH}_{4}$-hydrates should not exceed the maximum value of $14.81 \%$. The maximum mole fraction corresponds to fully occupied cavities by gas molecules in the water lattice of sI hydrate structure. We note that the original Yokozeki's $\mathrm{CH}_{4}$-hydrate model [11] did not fulfill this condition for temperatures above $316 \mathrm{~K}$.

\section{BinARY INTERACTION PARAMETERS For AQUEOUS SOlUTIONS}

The S-L-V EoS considers two sets of binary interaction parameters: one for the fluid phase, i.e. vapor-liquid equilibrium (VLE), and one for the hydrate phase, i.e. solid solution. The binary interaction parameter $k_{i j}$ in equation (6) is usually taken as temperature dependent for the aqueous solutions. The $k_{i j}$ parameter was obtained by correlating the S-L-V EoS to the available experimental data for the VLE and for the hydrate formation conditions.

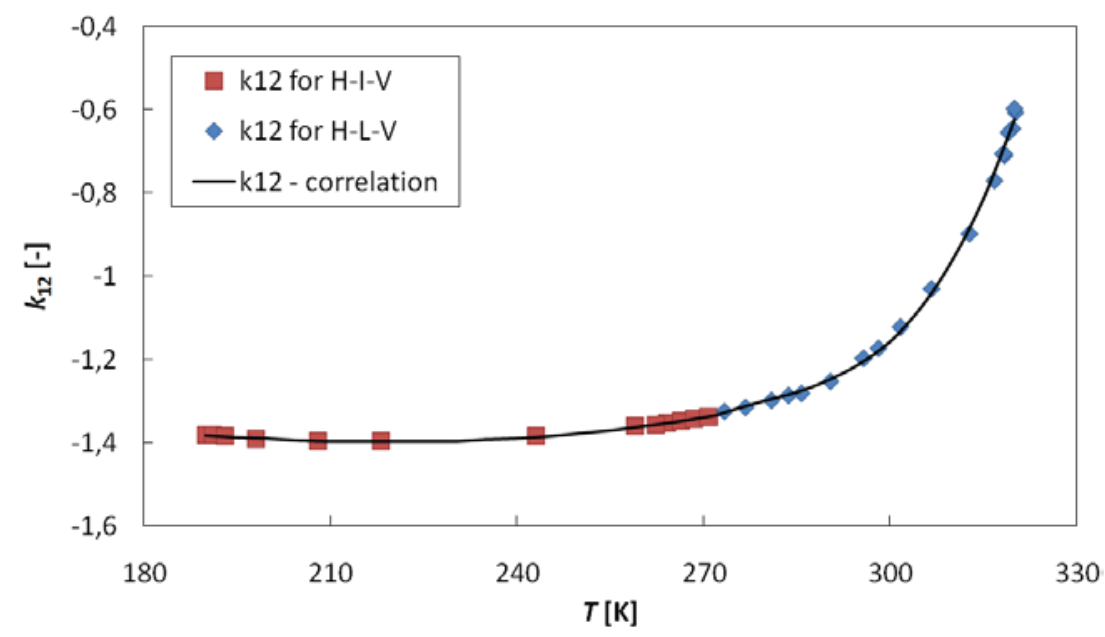

Figure 2: Binary interaction parameter $k_{12}$ for hydrate phase of $\mathrm{CH}_{4}(1)+\mathrm{H}_{2} \mathrm{O}(2)$ mixture; phases: $\mathrm{H}$ - hydrate, $\mathrm{L}$ - liquid, V - vapor, I - water ice

The fluid phases of the methane-water mixture were modeled with the same binary interaction parameter as by Yokozeki [11]. The $k_{i j}$ parameter was calculated from the following linear temperature function

$$
k_{i j}(T)=a_{0}+a_{1} T[\mathrm{~K}] .
$$


Since the hydrate phase was modeled in a different way than by Yokozeki [11] -see section 4- the $k_{i j}$ parameter for the solid phase had to be found separately. The modified S-L-V EoS was correlated to the experimental data points for the hydrate formation conditions, i.e. the three phase equilibrium lines at which the gas-hydrate is present, selected from the databases by Sloan and Koh [2] and Kroenlein et al. [20]. For brevity we do not provide full references for all experimental data considered in our study but only the abbreviations used by Sloan and Koh [2] and Kroenlein et al. [20]. The binary interaction parameter for the $\mathrm{CH}_{4}$-hydrate was fitted to the data by Roberts et al. (1940), Deaton and Frost (1946), Marshall et al. (1964), Falabella (1975), Adisasmito et al. (1991), Makogon and Sloan (1994), and Dyadin and Aladko (1996). Figure 2 shows the temperature dependence of the binary interaction parameter $k_{12}$ for $\mathrm{CH}_{4}$-hydrate; methane(1) and water(2). The parameter $k_{12}$ was correlated with the third-order polynomial function

$$
k_{12}(T)=a_{0}+a_{1} \tau+a_{2} \tau^{2}+a_{3} \tau^{3},
$$

where $\tau=T[\mathrm{~K}] / 200 \mathrm{~K}$. Table 2 summarizes values of the coefficients $a_{0}$ to $a_{3}$ for the binary interaction parameter for the VLE and hydrate phase of the methane-water binary mixture.

\begin{tabular}{lccccc}
\hline & $\mathrm{CH}_{4}+\mathrm{H}_{2} \mathrm{O}$ VLE & \multicolumn{3}{c}{$\mathrm{CH}_{4} \mathrm{H}$-phase } \\
& $k_{12}{ }^{*}$ & $k_{21}{ }^{*}$ & \multicolumn{2}{c}{$k_{12}$} & $k_{21}$ \\
& & & $T<272 \mathrm{~K}$ & $T \geq 272 \mathrm{~K}$ & \\
\hline$a_{0}$ & $-1.5097 \mathrm{E}+00$ & -0.0245 & $-1.1753335 \mathrm{E}+00$ & $-1.7930077 \mathrm{E}+02$ & -0.390 \\
$a_{1}$ & $4.0579 \mathrm{E}-03$ & - & $2.6636395 \mathrm{E}-01$ & $3.7992213 \mathrm{E}+02$ & - \\
$a_{2}$ & - & - & $-1.0397601 \mathrm{E}+00$ & $-2.7085558 \mathrm{E}+02$ & - \\
$a_{3}$ & - & - & $5.5719456 \mathrm{E}-01$ & $6.4499851 \mathrm{E}+01$ & - \\
\hline
\end{tabular}

* Taken from Yokozeki [11]

Table 2: Coefficients of the temperature correlation for the binary interaction parameter for the methane(1)-water(2) mixture

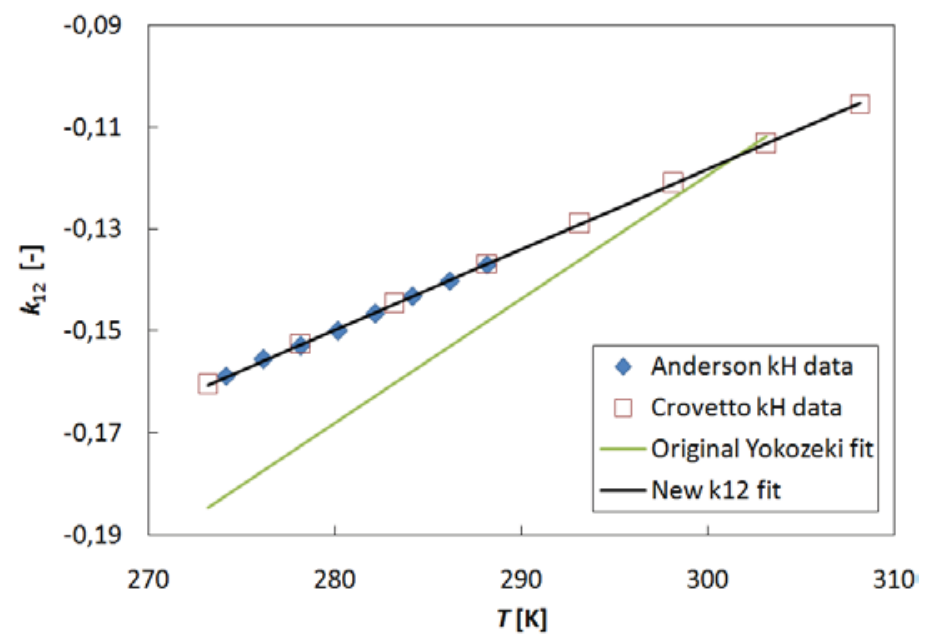

Figure 3: Binary interaction parameter $k_{12}$ for the VLE of $\mathrm{CO}_{2}(1)+\mathrm{H}_{2} \mathrm{O}$ (2) mixture determined from the solubility data [21],[22]

For the $\mathrm{CO}_{2}+\mathrm{H}_{2} \mathrm{O}$ system, the binary interaction parameters had to be correlated both for the VLE and the solid phase as another combination of the pure component 
parameters $a$ to $d$ were used for this mixture than by Yokozeki [10]. The $k_{12}$ parameter for VLE was correlated to the Henry's law data provided by Crovetto [21] and Anderson [22]. The Henry's law constant $k_{\mathrm{H}}$ was calculated from the following equation

$$
k_{\mathrm{H}}=\lim _{x_{1} \rightarrow 0}\left(\frac{f_{1}^{\mathrm{L}}}{x_{1}}\right)=\lim _{x_{1} \rightarrow 0}\left(\frac{\phi_{1}^{\mathrm{L}} x_{1} p}{x_{1}}\right)=p_{2}^{\mathrm{V}} \phi_{1}^{\mathrm{L}}\left(T, p_{2}^{\mathrm{V}}, x_{1}=0\right) ，
$$

where $p_{2}^{\mathrm{V}}$ states for the vapor pressure of the pure water at the given temperature. Figure 3 shows the binary interaction parameter $k_{12}$ determined for the modified S-L-V EoS. As can be seen, the new correlation differs from the original Yokozeki's dependence [10]. The $k_{21}$ parameter was determined from the experimental data by Wendland et al. [23] measured for the three phase equilibrium line $V-L_{1}-L_{2}\left(L_{1}-\right.$ carbon dioxide rich liquid). The binary interaction parameter for the hydrate phase was fitted to the various three phase equilibrium lines including $\mathrm{V}-\mathrm{H}-\mathrm{I}, \mathrm{V}-\mathrm{H}-\mathrm{L}_{1}, \mathrm{~V}-\mathrm{H}-\mathrm{L}_{2}$, and $\mathrm{H}-\mathrm{L}_{1}-\mathrm{L}_{2}$ equilibrium. The experimental data for the $\mathrm{CO}_{2}$-hydrate formation conditions were again taken from the databases by Sloan and Koh [2] and Kroenlein et al. [20]. Following data sets were considered in our study: Deaton and Frost (1946), Larson (1955), Takenouchi and Kennedy (1965), Vlahakis et al. (1972), Falabella (1975), Ng and Robinson (1985), Ohgaki et al. (1993), Nakano et al. (1998b), Wendland (1999), Moojier-van den Heuvel et al. (2001), Hachikubo et al. (2002), and Mohammadi et al. (2005).

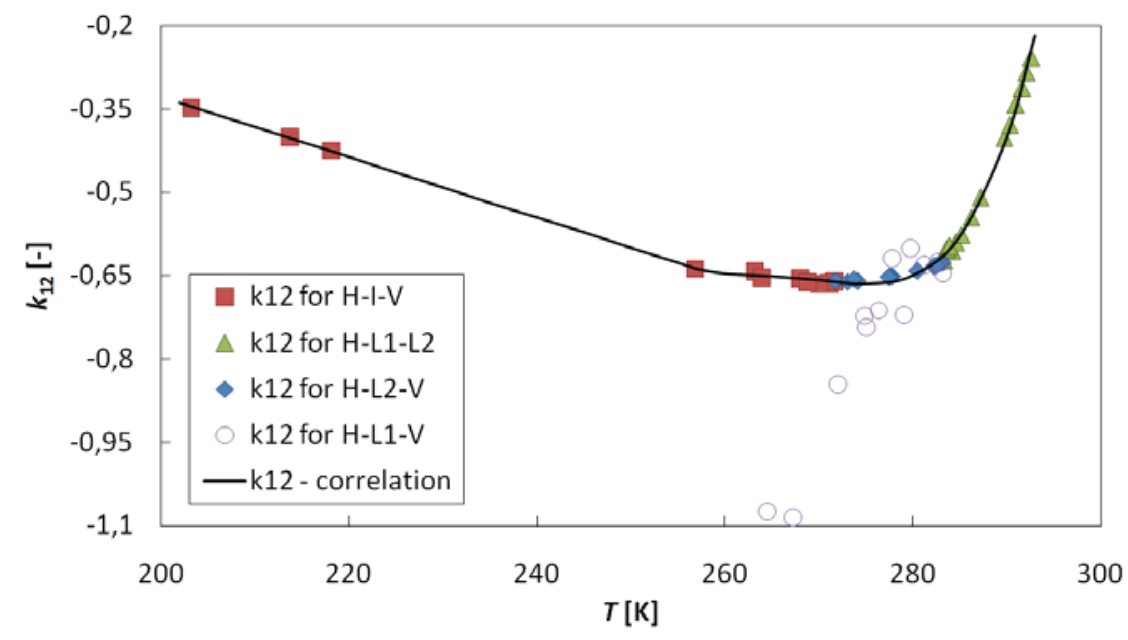

Figure 4: Binary interaction parameter $k_{12}$ for hydrate phase of $\mathrm{CO}_{2}(1)+$ $\mathrm{H}_{2} \mathrm{O}(2)$ mixture; phases: $\mathrm{H}$ - hydrate, I - water ice, $\mathrm{L1}-\mathrm{CO}_{2}$-rich liquid, $\mathrm{L2}-\mathrm{H}_{2} \mathrm{O}$-rich liquid, $\mathrm{V}$ - vapor

\begin{tabular}{cccccc}
\hline \multicolumn{4}{c}{$\mathrm{CO}_{2}+\mathrm{H}_{2} \mathrm{O}$ VLE } & \multicolumn{3}{c}{$\mathrm{CO}_{2} \mathrm{H}-$ phase } \\
& $k_{12}$ & $k_{21}$ & & $k_{12}$ & $k_{21}$ \\
& & & $T<257 \mathrm{~K}$ & $T \geq 257 \mathrm{~K}$ & \\
\hline$a_{0}$ & $-5.9126 \mathrm{E}-01$ & $-1.3961 \mathrm{E}+00$ & $7.5732474 \mathrm{E}-01$ & $5.61702434 \mathrm{E}+03$ & -0.095 \\
$a_{1}$ & $1.5768 \mathrm{E}-03$ & $4.1892 \mathrm{E}-03$ & $-1.0854259 \mathrm{E}+00$ & $-1.68464229 \mathrm{E}+04$ & - \\
$a_{2}$ & - & - & - & $1.89425800 \mathrm{E}+04$ & - \\
$a_{3}$ & - & - & - & $-9.46522357 \mathrm{E}+03$ & - \\
$a_{4}$ & - & - & - & $1.77333268 \mathrm{E}+03$ & - \\
\hline
\end{tabular}

Table 3: Coefficients of the temperature correlation for the binary interaction parameter for the carbon dioxide(1)-water mixture(2) 
Figure 4 shows the $k_{12}$ parameter depending on temperature. The data for the $\mathrm{V}-\mathrm{H}-\mathrm{L}_{1}$ equilibrium are rather inconsistent and lie aside from the other three phase lines. These data were therefore not used in the $k_{12}$ correlation. As it is shown in the following section, neglecting the $k_{12}$ parameter for $\mathrm{V}-\mathrm{H}-\mathrm{L}_{1}$ equilibrium did not result in any significant loss of accuracy of the modified S-L-V EoS. The parameter $k_{12}$ for the $\mathrm{CO}_{2}$-hydrate phase was correlated with the fourth-order polynomial function

$$
k_{12}(T)=a_{0}+a_{1} \tau+a_{2} \tau^{2}+a_{3} \tau^{3}+a_{4} \tau^{4},
$$

where again $\tau=T[\mathrm{~K}] / 200 \mathrm{~K}$. Values of the coefficients $a_{0}$ to $a_{4}$ for the binary interaction parameter for the VLE and hydrate phase of the carbon dioxide-water system are listed in Table 3.

\section{RESULTS}

The hydrate formation conditions predicted by the modified S-L-V EoS were compared with the experimental data. Figure 5 shows the $\log (p)-T$ diagram for the methane-water mixture. As can be seen, the EoS achieved quite good agreement with experiments over wide temperature and pressure ranges of $190 \div 318 \mathrm{~K}$ and $0 \div 400 \mathrm{MPa}$, respectively.

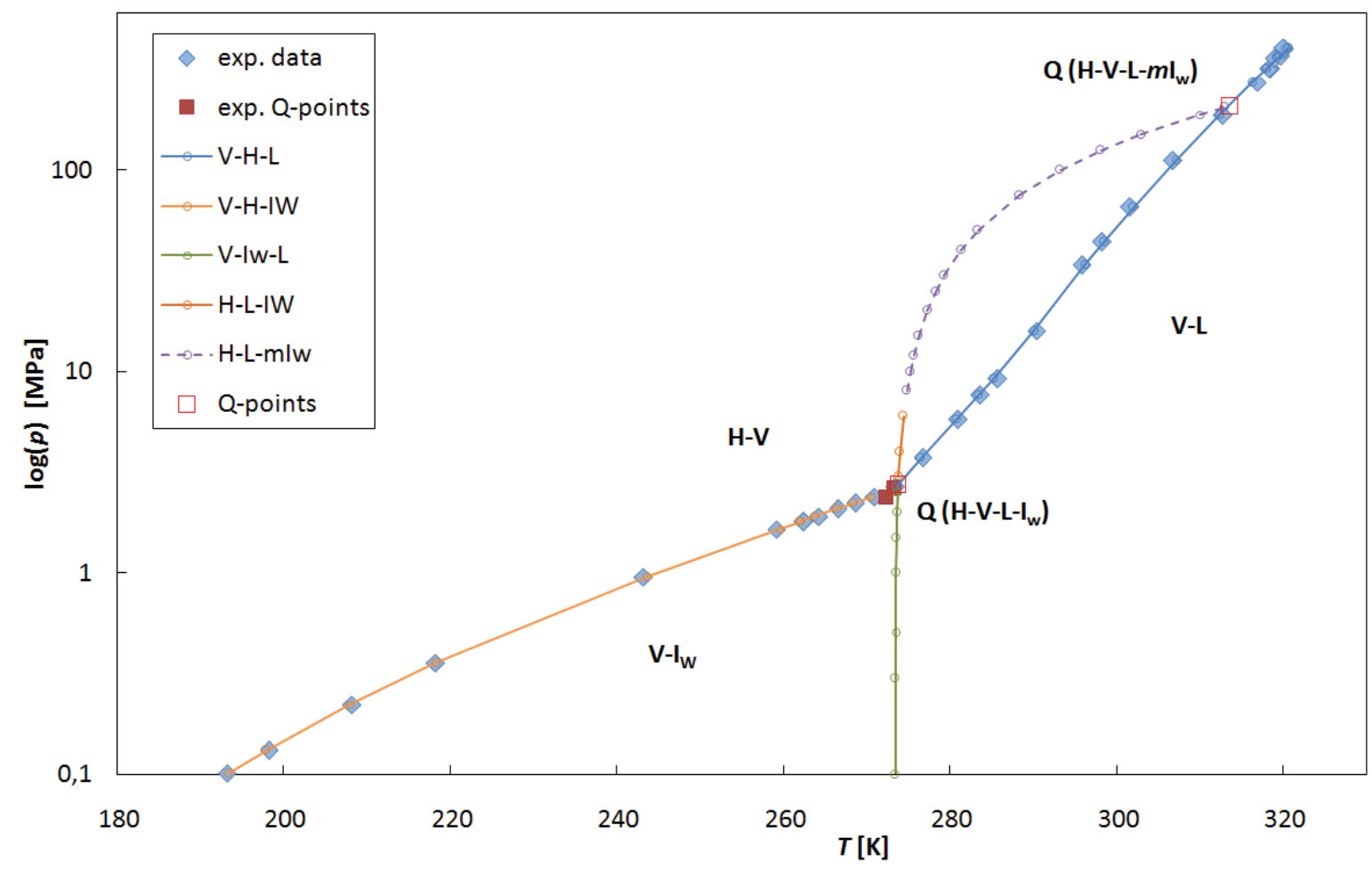

Figure 5: Phase diagram of the water-methane mixture. Comparison of selected experimental data with results of the modified S-L-V EoS

Like the original S-L-V EoS by Yokozeki [11], the modified EoS predicted two different quadruple points. The experimentally verified $\mathrm{V}-\mathrm{H}-\mathrm{L}-\mathrm{I}$ point at $T=273.74 \mathrm{~K}$ and $p=2.744 \mathrm{MPa}$ and the second theoretical $\mathrm{V}-\mathrm{H}-\mathrm{L}-m \mathrm{I}$ point at the high pressure $208.4 \mathrm{MPa}$ and temperature $313.5 \mathrm{~K}$. The pure water ice exists only to a maximum pressure of approximately $6.0 \mathrm{MPa}$ [11]. The three phase equilibrium line $\mathrm{H}-\mathrm{L}-\mathrm{mI}$ calculated at high pressures is therefore considered as the metastable equilibrium with the metastable water ice $(m \mathrm{I})$. However as far as we know, the second quadruple point V-H-L-mI was 
not experimentally verified and the dashed three-phase line H-L-mI should therefore be considered only as a hypothetical solution.

The predicted mole fraction of methane in the hydrate phase varying with temperature and pressure is plotted in Figure 6. The data points were calculated at the experimental temperatures and pressures of the various three-phase equilibrium lines. The mole fraction of $\mathrm{CH}_{4}$ did not exceed the physically defined maximum value of $14.81 \%$.
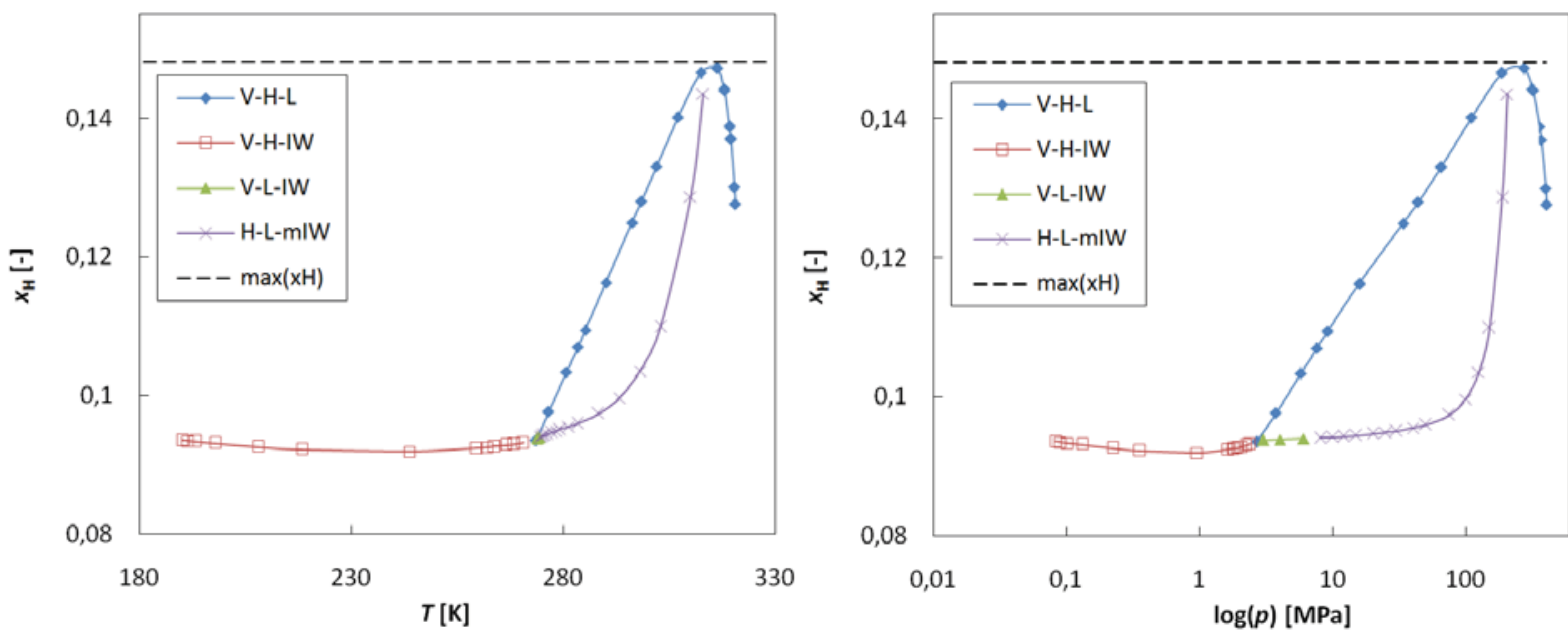

Figure 6: Mole fraction of methane in the gas-hydrate along four different three-phase equilibrium lines predicted by the modified S-L-V EoS

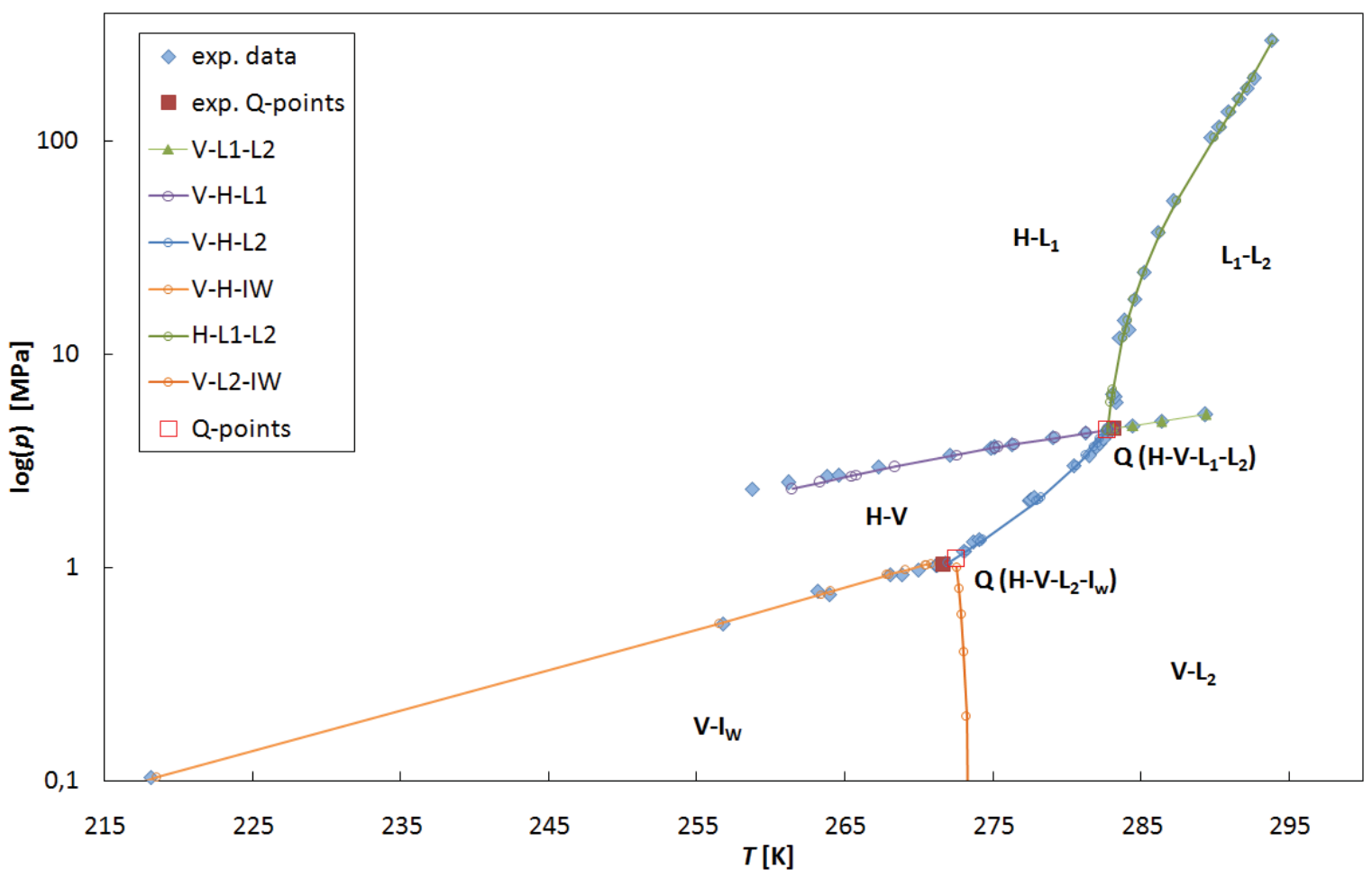

Figure 7: Phase diagram of the water-carbon dioxide mixture. Comparison of selected experimental data with results of the modified S-L-V EoS

Figure 7 shows the $\log (p)-T$ diagram for the carbon dioxide-water system. The modified $\mathrm{S}-\mathrm{L}-\mathrm{V}$ Eos is again in a good agreement with the experimental hydrate formation data in 
this case. The ranges of validity are $220 \div 294 \mathrm{~K}$ in temperature and $0 \div 200 \mathrm{MPa}$ in pressure. The quadruple points were predicted at the pressures of $1.109 \mathrm{MPa}$ and $4.438 \mathrm{MPa}$ and temperatures of $272.50 \mathrm{~K}$ and $282.73 \mathrm{~K}$ for $\mathrm{V}-\mathrm{L}_{2}-\mathrm{H}-\mathrm{I}$ and $\mathrm{V}-\mathrm{L}_{1}-\mathrm{L}_{2}-\mathrm{H}$, respectively. Both these results are comparable with the experimental values [2].
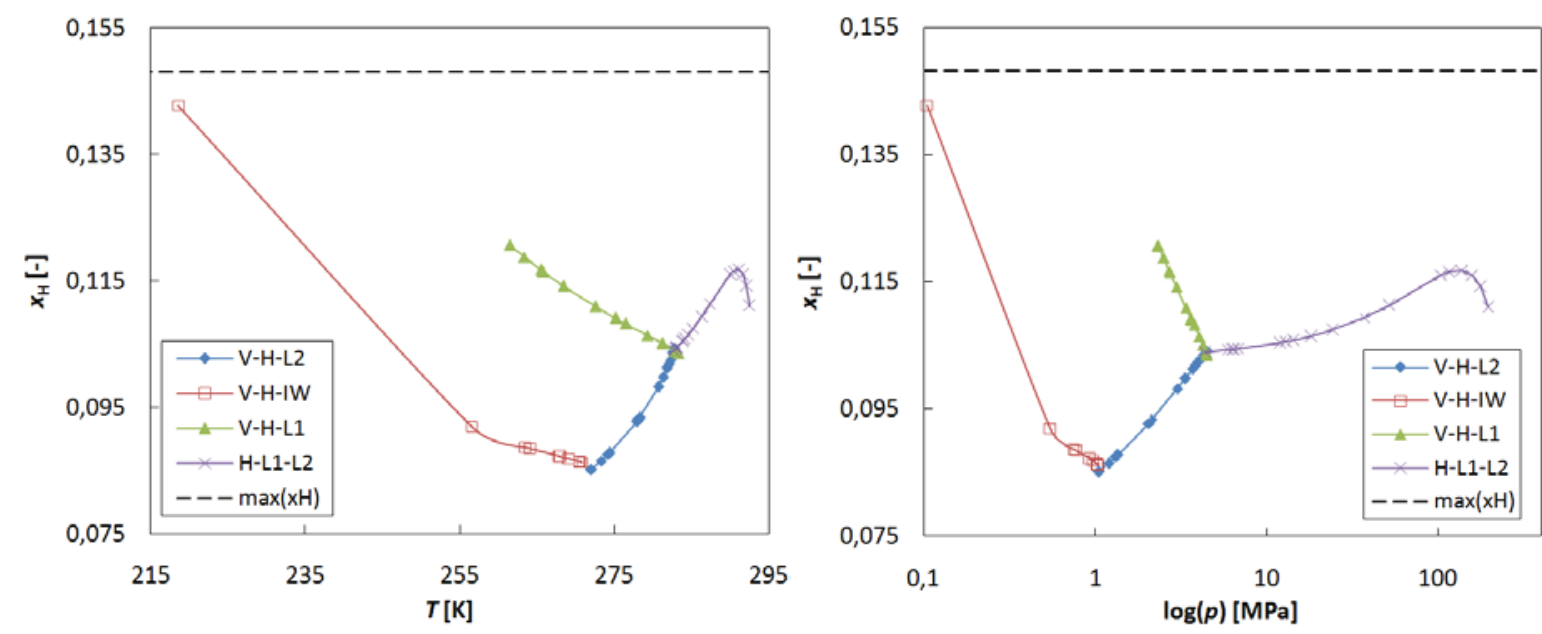

Figure 8: Mole fraction of carbon dioxide in the gas-hydrate along four different three phase equilibrium lines predicted by the EoS

The predicted mole fraction of carbon dioxide in the hydrate phase did not exceed the maximum allowed value at any of the four three-phase lines; see Figure 8 . The average predicted value is around $10.5 \%$ which approximately corresponds to the situation when only the large cavities of the water lattice are occupied. This result seems rather reasonable as the $\mathrm{CO}_{2}$ molecules are relatively large. It is therefore quite probable that the small cavities are occupied only marginally.

\section{CONCLUSION}

The analytical S-L-V EoS by Yokozeki [9]-[11] was examined for prediction of the gashydrate formation conditions in this study. The EoS was modified to improve its predictive ability for the $\mathrm{CH}_{4}$ - and $\mathrm{CO}_{2}$-hydrates. The hydrate phase was modeled by increasing the water attraction parameter compared to the pure water ice. Binary interaction parameters for the VLE and the hydrate phase were correlated to relatively large amount of the phase equilibrium experimental data. The computational algorithm allows solving various types of the phase equilibria using the Newton-Raphson method applied on the condition of equal fugacities. The modified S-L-V EoS achieved quite good accuracy and can be used as an initial estimate for the hydrate formation conditions. The predicted mole fraction of gas in the hydrate phase did not exceed the maximum value of $14.81 \%$ in the considered temperature and pressure ranges.

\section{NOMENCLATURE}

$a \ldots$ attraction parameter $\left[\mathrm{J} \cdot \mathrm{m}^{3} \cdot \mathrm{mol}^{-2}\right]$

$a_{0} \div 4 \ldots$ coefficient

$b, c, d \ldots$ volumetric param. $\left[\mathrm{m}^{3} \cdot \mathrm{mol}^{-1}\right]$

$e$... general parameter $\left[\mathrm{m}^{3} \cdot \mathrm{mol}^{-1}\right]$

$c_{a} \ldots$ correction coefficient $[-]$

$f$... fugacity $[\mathrm{Pa}]$

$k_{i j} \ldots$ binary interaction parameter [-]

$k_{\mathrm{H}} \ldots$ Henry's law constant [Pa] 


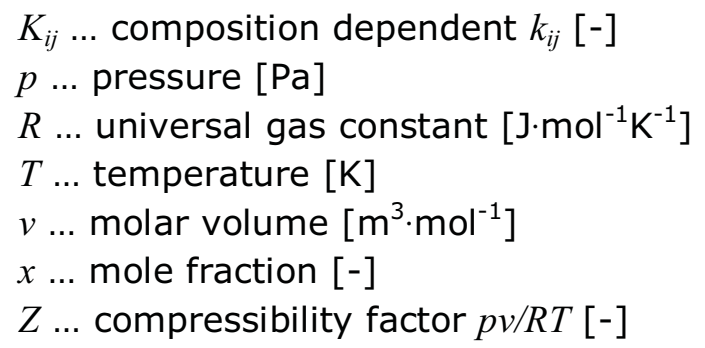

\author{
Subscripts \\ c ... critical point condition \\ $i, j \ldots$ index of a component \\ $\mathrm{r}$... reduced quantity
}

Superscripts
.... reduced quantity
H ... gas-hydrate
I ... ice
L ... liquid
V ... vapor

\section{ACKNOWLEDGEMENTS}

The authors are grateful to the International Association for the Properties of Water and Steam (IAPWS) which supported Václav Vinš's stay at the Ruhr University in Bochum. The project has also been partly supported by the Czech Science Foundation grants No. P101/11/P046, P101/11/1593, and by the Research Plan of the Institute of Thermomechanics AS ČR, v.v.i. No. AV0Z20760514.

\section{REFERENCES}

[1] Van der Waals J.H. and Platteeuw J.C.: Clathrate solutions, Advan. Chem. Phys. 2, 1959, 1-57

[2] Sloan E.D. and Koh C.A.: Clathrate hydrates of natural gases, 3rd ed., CRC Press, Taylor \& Francis group, 2008

[3] Parrish W.R. and Prausnitz J.M.: Dissociation pressure of gas hydrates formed by gas mixtures, Ind. Eng. Chem. Process Des. Develop. 11, 1972, 26-35

[4] Lee S.Y. and Holder G.D.: Model for gas hydrate equilibria using a variable reference chemical potential: Part 1, AIChE J. 48, 2002, 161-167

[5] Klauda J.B. and Sandler S.I.: Phase behavior of clathrate hydrates: a model for single and multiple gas component hydrates, Chem. Eng. Sci. 58, 2003, 27-41

[6] Bandyopadhyay A.A. and Klauda J.B.: Gas hydrate structure and pressure predictions based on an updated fugacity-based model with the PSRK equation of state, Ind. Eng. Chem. Res. 50, 2011, 148-157

[7] Ballard A.L. and Sloan Jr. E.D.: The next generation of hydrate prediction: An Overview, J. Supermolec. Chem. 2, 2002, 385-392

[8] Ballard A.L. and Sloan Jr. E.D.: The next generation of hydrate prediction I. Hydrate standard states and incorporation of spectroscopy, Fluid Phase Equil. 194-197, 2002, 371-383

[9] Yokozeki A.: Analytical equation of state for solid-liquid-vapor Phases, International Journal of Thermophysics 24, 2003, 589-620

[10] Yokozeki A.: Solid-liquid-vapor phases of water and water-carbon dioxide mixtures using a simple analytical equation of state, Fluid Phase Equil. 222-223, 2004 55-66

[11] Yokozeki A.: Methane gas hydrates viewed through unified solid-liquid-vapor equations of state, International Journal of Thermophysics 26, 2005, 743-765 
[12] Wagner W. and Pruss A.: The IAPWS formulation 1995 for the thermodynamic properties of ordinary water substance for general and scientific use, J. Phys. Chem. Ref. Data 31, 2002, 387-535

[13] Span R. and Wagner W.: A new equation of state for carbon dioxide covering the fluid region from the triple-point temperature to $1100 \mathrm{~K}$ at pressures up to $800 \mathrm{MPa}$, J. Phys. Chem. Ref. Data 25, 1996, 1509-1596

[14] Span R. and Gernert J.: Accurate property models for application in CSS processes, Proc. International Conference on Applied Energy, 21-23 April 2010, Singapore

[15] IAPWS: Revised Release on the Equation of State 2006 for $\mathrm{H} 2 \mathrm{O}$ Ice Ih, The International Association for the Properties of Water and Steam, (September 2009), available at http://www.iapws.org/

[16] Jäger A. and Span R.: Equation of state for solid carbon dioxide in form of the Gibbs free energy. accepted for publication in Proc. Asian Thermophys. Prop. Conf. 2010, Beijing (2010)

[17] Van der Waals J.D.: On the continuity of the gaseous and liquid states, Doctoral Dissertation, 1873, Universiteit Leiden, Holland

[18] Peng D.Y. and Robinson D.B.: A new two-constant equation of state, Ind. Eng. Chem. Fund. 15, 1976, 59-64

[19] Sengers J.V., Kayser R.F., Peters C.J., White H.J.: Equations of state for fluids and fluid mixtures, Experimental Thermodynamics, Volume 5, 1st ed., Elsevier Science B.V. (2000)

[20] Kroenlein K., Muzny C.D., Kazakov A., Diky V.V., Chirico R.D., Frenkel M., Sloan E.D.: Clathrate hydrate physical property database and web dissemination channel, J. Res. Natl. Inst. Stand. Technol. 115 (2010) 85-112, In http://gashydrates.nist.gov/

[21] Crovetto R.: Evaluation of solubility data of the system $\mathrm{CO}_{2}-\mathrm{H}_{2} \mathrm{O}$ from $273 \mathrm{~K}$ to critical point of water, J. Phys. Chem. Ref. Data 20, 1991, 575-589

[22] Anderson G.K.: Solubility of carbon dioxide in water under incipient clatharte formation conditions, J. Chem. Eng. Data 47, 2002, 219-222

[23] Wendland M., Hasse H., Maurer G.: Experimental pressure-temperature data on three- and four-phase equilibria of fluid, hydrate, and ice phases in the system carbon dioxide-water, J. Chem. Eng. Data 44, 1999, 901-906 